\title{
Review
}

\section{A comprehensive review of hypertrophic cardiomyopathy and arterial thromboembolism in cats}

\author{
Donghyun $\mathrm{Han}^{1}$ and Dong-In Jung ${ }^{2^{*}}$ \\ ${ }^{1}$ Section of Veterinary Research and Academic Consulting, Veteran, Inc., Seoul 06254, Korea \\ ${ }^{2}$ Institute of Animal Medicine, College of Veterinary Medicine, Gyeongsang National University, Jinju 52828, Korea
}

The number of cats requiring treatment for hypertrophic cardiomyopathy (HCM) and arterial thromboembolism (ATE) continues to increase, and the knowledge regarding its management is constantly evolving. The pathological lesions of HCM include hypertrophy of the left ventricle, which causes abnormalities in the relaxation function of the heart. This phenomenon increases the stiffness of the ventricular muscle, thereby reducing the ability of the left ventricle to fill with blood during diastole. This is accompanied by an increase in ventricular filling pressure and left atrial pressure. HCM in cats is characterized by concentric hypertrophy and atrial enlargement. Hypertrophic obstructive cardiomyopathy (HOCM) also involves a narrowed left ventricular outflow tract, and in humans, it is generally perceived to be a more serious disease. However, unlike in humans, HCM and HOCM in cats do not result in significantly different survival times. Heart murmurs, gallop rhythms, arhythmias, cardiac hypertrophy, congestive heart failure (CHF), ATE, and cardiac sudden death (CSD) have all been associated with HCM. Although the presence of a heart murmur is a characteristic feature of heart disease, it may be a functional one, which is defined as "dynamic right ventricular outflow track obstruction" (DRVOTO) in cats. Therefore, it is difficult to evaluate the presence of HCM based on the existence of a heart murmur alone. ATE typically affects one or both hind limbs, resulting in acute paralysis and severe pain, consistent with lower motor neuron disease. The clot, which is formed in the left atrium of the heart, travels to an artery and becomes an ATE, which then blocks the blood flow and impairs circulation, causing infarction. Therefore, ATE in cats is a serious condition. This review describes the results of the latest research on HCM and ATE, the most common heart conditions in cats.
Key words: hypertrophic cardiomyopathy, arterial thromboembolism, heart murmur, congestive heart failure, cat

\section{Introduction}

Hypertrophic cardiomyopathy (HCM) is a common type of heart disease affecting cats, and is characterized by concentric hypertrophy and atrial enlargement. Heart murmurs, gallop sounds, arrhythmias, cardiac hypertrophy, congestive heart failure (CHF), arterial thromboembolism (ATE), and cardiac sudden death (CSD) have all been associated with HCM. CHF may occur secondary to stress, corticosteroid therapy, anesthesia, or intravenous fluid therapy in feline HCM patients. HCM in cats may be either a primary (idiopathic, hereditary) or secondary disease. Therefore, in order to accurately diagnose HCM, it is necessary to rule out such concurrent diseases as hyperthyroidism, systemic hypertension, and aortic stenosis.

Although the presence of a heart murmur is a characteristic feature of heart disease, it may be a functional one, defines as dynamic right ventricular outflow track obstruction (DRVOTO) in cats. The specificity and sensitivity of heart murmurs in the diagnosis of HCM are $31 \%$ and $87 \%$, respectively. Therefore, it is difficult to diagnose HCM based on the presence of a heart murmur alone, and in fact, $69 \%$ of cats with HCM do not have a heart murmur at all [1]. According to a study by Rishniw et al., out of a group of cats over 4 years of age that had been diagnosed with DRVOTO heart murmurs, only $27 \%$ had evidence of heart disease [2]. The heart murmur present with DRVOTO may be caused by the high cardiac output associated with hyperthyroidism, anemia, inflammatory disease, or chronic kidney disease, regardless of the presence of systemic hypertension.

Studies regarding the genetic propensity for HCM have

*Corresponding author: Dong-In Jung

Institute of Animal Medicine, College of Veterinary Medicine, Gyeongsang National University, Jinju 52828, Korea

Tel: +82-55-772-2361, E-mail: jungdi@gnu.ac.kr 
identified a mutation in the Myocardial Binding Protein C3 (MYBPC3) gene, but this is not present in all cats with HCM. Certain breeds are predisposed to the development of HCM, among these are the Ragdoll, Persian, Himalayan, Birman, Bengal, Sphinx, and Maine Coon. This condition is especially severe in Ragdoll cats, being diagnosed at approximately 15 months of age, on average [3]. In spite of this, the domestic shorthair cat remains the most commonly diagnosed breed with HCM in clinical trials.

The pathological lesions of HCM include abnormal hypertrophy of the left ventricle. This causes abnormalities in the heart's ability to relax. This phenomenon increases the stiffness of the ventricular muscle, thereby reducing the ability of the left ventricle to fill with blood during diastole. This is accompanied by an increase in ventricular filling pressure and left atrial pressure. It also induces an increase in pulmonary vein pressure, with pulmonary edema occurring at pressures greater than $25 \mathrm{mmHg}[4,5]$.

ATE typically affects one or both hind limbs, resulting in acute paralysis and severe pain, consistent with lower motor neuron disease. Both hind limbs are affected $71 \%$ of the time [6], with a blood clot obstructing flow to the limbs (usually via the iliac arteries). The affected hind limbs feel cold, with absent pulses and cyanotic paw pads. In patients with suspected HCM, it is useful to check for the presence of femoral pulses using Doppler blood pressure monitoring.

ATE occurs frequently in cats with HCM [7, 8]. Thrombosis, the cause of ATE, occurs commonly in the left atrium and rarely in the right heart and left ventricle [7, 8]. The clot, which is formed in the left atrium, travels to an artery becoming an ATE, which then blocks blood flow and impairs circulation, causing an infarction. Therefore, ATE in cats creates a grave situation.

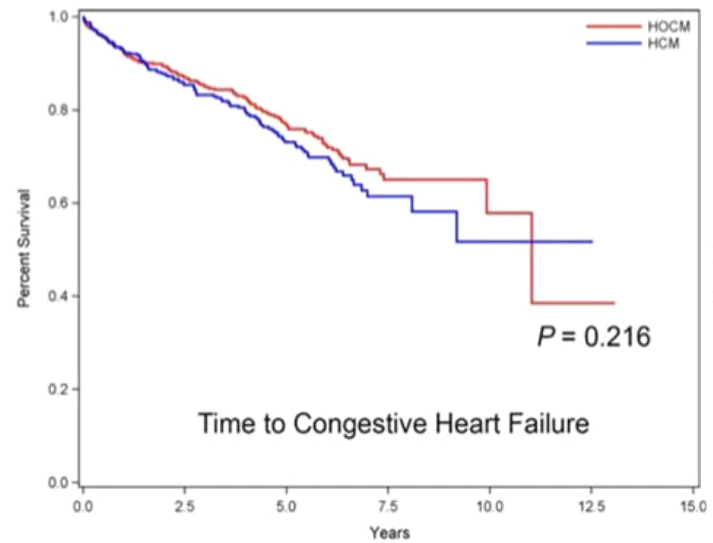

\section{Large-scale cohort study of preclinical HCM}

A large-scale cohort study evaluating cats with asymptomatic HCM, the REVEAL study, was conducted over an approximately 15 year period (from October 2001 to January 2016) [9]. The study by Fox et al. included 430 asymptomatic HCM patients, 578 asymptomatic hypertrophic obstructive cardiomyopathy (HOCM) patients, and 722 healthy cats as controls from a total of 54 centers in 21 countries within the US and Europe. At the completion of the study, patients were assigned to the CHF, ATE, or CSD groups. The breadth of this study confirms the interest that veterinary cardiologists have in preclinical HCM in cats.

Out of the 1,008 combined HCM and HOCM patients, $30.5 \%$ were diagnosed with $\mathrm{CHF}$, ATE, or both. In addition, the cardiac mortality rate was found to be $27.9 \%$. The resultant risk for the development of $\mathrm{CHF}$ and $\mathrm{ATE}$ in patients with HCM and HOCM was calculated to be $7.0 \%$ and $3.5 \%$ in the first year of life, $19.9 \%$ and $9.7 \%$ in the fifth year of life, and $23.9 \%$ and $11.3 \%$ in the tenth year of life, respectively. The risk of death was $6.7 \%$ in the first year of life, $22.8 \%$ in the fifth year of life, and $28.3 \%$ in the tenth year of life.

These results imply that in asymptomatic HCM and HOCM patients, the risk of CHF, ATE, CSD is agerelated. One of the most important findings in this study is that although HOCM, which narrows the left ventricular outflow tract, is generally perceived as a more serious condition than HCM in human medicine [10, 11], no significant difference in survival was found between cats affected with the two conditions (Fig. 1).

\section{Diagnostic technique for $\mathrm{HCM}$}

In order to identify cats affected with HCM in clinical

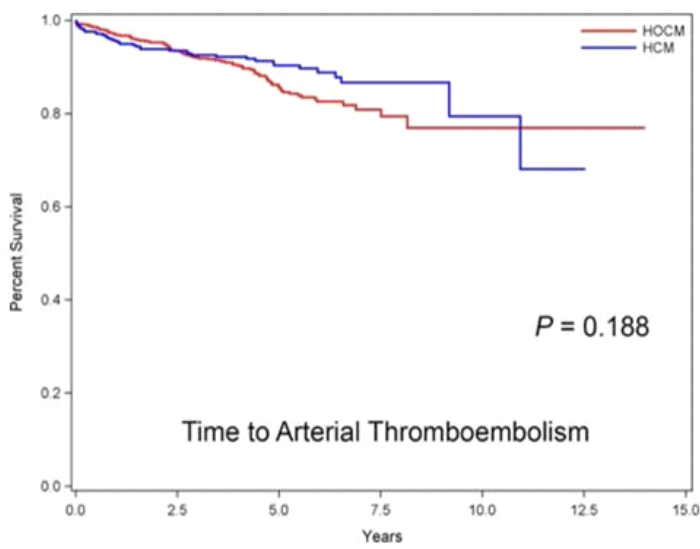

Fig. 1. REVEAL study. Comparison of survival times between cats with HCM or HOCM and concurrent congestive heart failure or arterial thrombosis. No significant difference was identified $(p=0.216 / p=0.188)$. HCM, hypertrophic cardiomyopathy; HOCM, hypertrophic obstructive cardiomyopathy. Adapted from J Vet Intern Med 2018;32:930-943 [9]. 
practice, diagnostics are required to assess the patient's condition and to determine the severity of the disease. Typical diagnostics include chest radiographs, electrocardiogram (ECG), NT-proBNP, echocardiography, blood pressure, and total T4 measurement if the patient is over 7 years old.

HCM is a disease manifested by hypertrophy of the myocardium and congestion of the atrium. Thoracic radiography is very important in the evaluation of cats with suspected HCM. The normal values for the VHS (Vertebral Heart Score), which compares the size of the heart to the length of the vertebrae, are $7.5 \pm 0.3$ [12]. An increasing VHS value suggests that the heart is becoming enlarged. However, thoracic radiography is not a very specific test for the diagnosis of HCM, and it is not possible to diagnose ATE in this manner. In addition, in early HCM, the appearance of the heart on radiographs is normal. In order to identify CHF caused by HCM, it is necessary to identify such characteristic features as left atrial enlargement, pulmonary vein engorgement, pleural effusion, alveolar or interstitial pulmonary infiltration or pulmonary edema occurring primarily in the caudal lung lobes.

The ECG is not overly sensitive or specific for the diagnosis of HCM. This test is used mainly to identify the arrhythmias that may occur in cats with HCM, namely ventricular premature beats, ventricular tachycardia, atrial premature beats, atrial tachycardia, and atrial fibrillation

The NT-proBNP test is widely used as an early screening method for the diagnosis of heart disease in cats. Brain natriuretic peptide (BNP) causes the excretion of sodium into the urine, and its level increases when stress or hemodynamic change occurs within the ventricle. Increased BNP levels result in the expansion of blood vessels, reduced activity of the RAAS, and enhanced diuresis caused by the excretion of sodium. If the NT-proBNP level is elevated in a cat with pulmonary disease, this suggests heart disease that is a possible cause. It has been reported that the NT-proBNP level is elevated even in cats with occult HCM [13]. However, although the NT-proBNP test is a very easy and useful diagnostic modality, the levels may also be elevated with kidney disease, hypertension (systemic or pulmonary), infection, or endocrine disease. In fact, a study comparing NT-proBNP levels in cats with hyperthyroidism to those with HCM did not reveal a significant difference in the values [14]. Veterinary cardiologists have been trained to consider the NT-proBNP test as only a part of the diagnostic work-up for heart disease.

Echocardiography is useful for the morphologic and functional evaluation of the heart in cats with HCM, which is characterized by hypertrophy of the ventricles. The most important findings during cardiac ultrasound include enlargement of the left ventricle, enlargement of the left atrium, fibrosis of the myocardium, degree of left ventricular relaxation and contraction, and evidence of thrombus formation.

\section{ATE as a major complication}

Complications of HCM include CHF, ATE, and CSD. To assess the risk of thrombosis, one needs to identify abnormal contrast known as "smoke" within the atria and
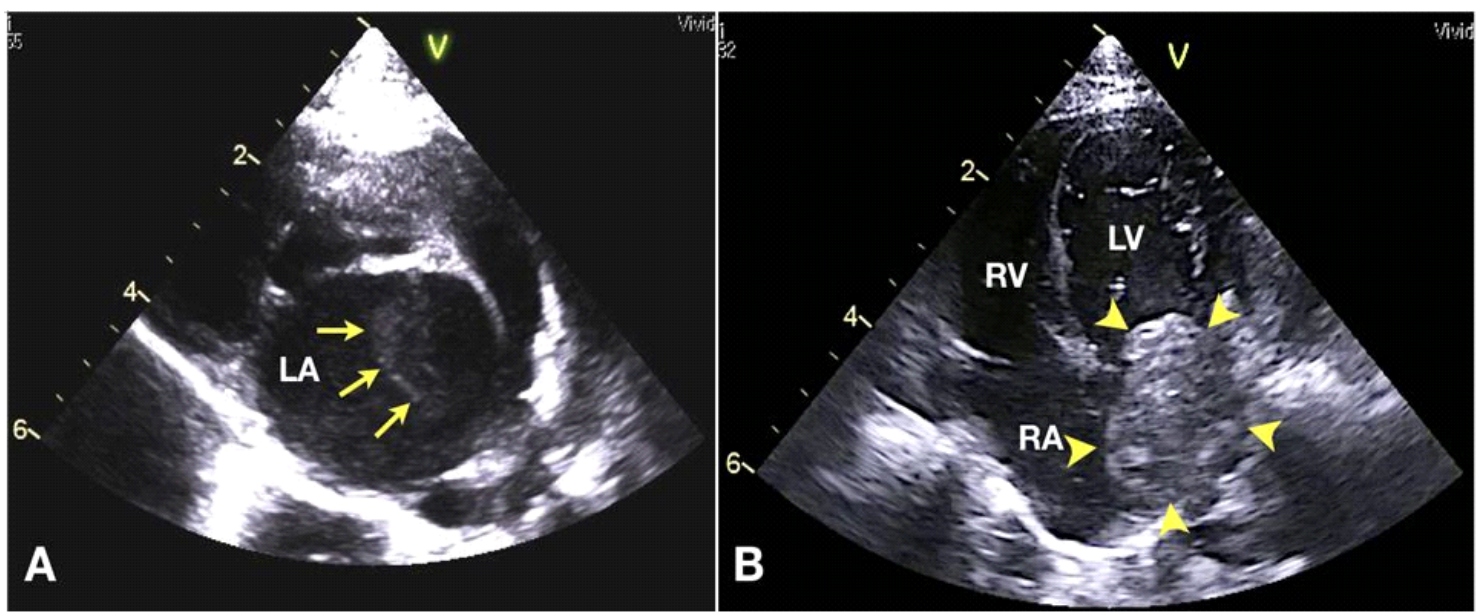

Fig. 2. (A) An HCM patient with "smoke" identified on an echocardiographic image. The presenting clinical sign in this patient was hind limb paralysis. Echocardiography revealed an enlarged left atrium (LA) and a suspected thrombus in the LA ("smoke", yellow arrow). (B) Thrombus in the LA. The patient was presented with severe pain and hind limb paralysis. Echocardiography was used to diagnose ATE. The patient was treated aggressively, but unfortunately did not respond to treatment and was ultimately euthanized. Echocardiography revealed enlarged left and right atria as well as a thrombus in the LA (yellow arrow head). HCM, hypertrophic cardiomyopathy; ATE, arterial thromboembolism. 
an enlarged left atrium on the echocardiogram (Fig. 2A). A thrombus may be visualized in the left atrium (Fig. 2B).

Recently, a study of 250 cats with ATE over an approximately 8 year period (from 2004 to 2012) from three animal hospitals in the UK was presented in the Journal of Veterinary Internal Medicine [15]. According to the study, 153 cats $(61.2 \%)$ were euthanized and only 6 cats survived for more than 12 months. Of the 97 cats that were not euthanized, 68 (70.1\%) survived for more than 24 hours following the onset of symptoms, and 30 of the $68(44.1 \%)$ survived for 7 days. The average survival time of the cats in the study was 94 days (range 42-164 days; Fig. 3). The results of this study indicate that ATE in cats is associated with very high morbidity and mortality rates, and that a poor prognosis exists even for the survivors.

The thrombi associated with ATE can theoretically block any blood vessel, resulting in infarction, but rarely occur in the brachial, renal, splanchnic, and cerebral arteries $[6,16]$. Most thrombi are located at the bifurcation of the distal aorta where it divides into the iliac arteries, which supply blood to the hind limbs [6]. A thrombus developing in this area is called a "saddle thrombus," named so because the morphological features of the vessels in this area resemble the saddle of a horse.

A saddle thrombus blocks the blood supply to one or both of the iliac arteries, thereby compromising the blood supply to the hind limbs, as well as causing the release of inflammatory mediators such as serotonin and prostaglandin. This causes the muscles of the hind limbs to stiffen, the limbs to feel cold when touched, the paw pads to become discolored, and the hind limbs to become paralyzed, resulting in sudden collapse and vocalization.

While all cats have the potential to develop ATE such

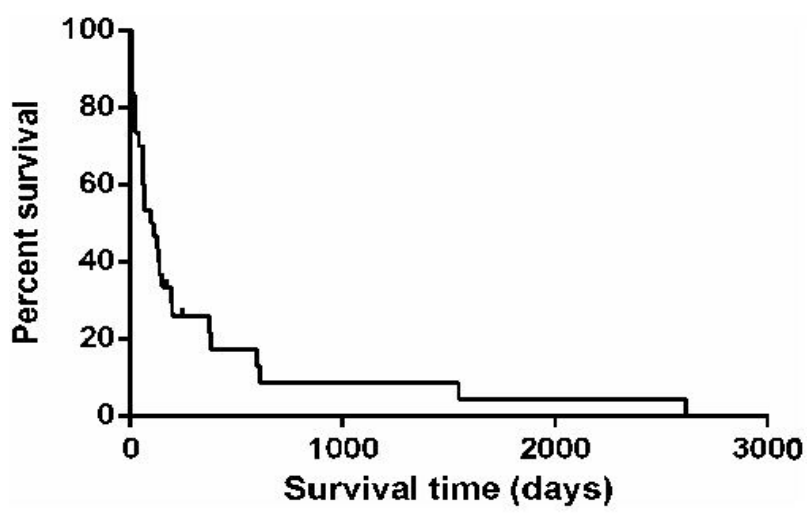

Fig. 3. Kaplan-Meier survival curve for cats surviving at least 7 days after the diagnosis of ATE. Mean survival time was 94 days (42-164 days). ATE, arterial thromboembolism. Adapted from J Vet Intern Med 2014;28:102-108 [15]. breeds as the Abyssinian, Ragdoll, and Birman are overrepresented. Male cats more commonly affected by both HCM and ATE than female cats $[17,18]$.

\section{Risk factors for ATE}

Left atrial dilatation occurs in $93 \%$ of cats with ATE [6], and echocardiography is a useful screening method for predicting the risk of ATE formation. The maximum diameter of the left atrium is measured from the long axis of the heart on the right parasternal 4-chamber image, resulting in a sagittal view. The left atrial measurement is obtained at the end of ventricular systole, parallel to the posterior wall of the left atrium. A useful tip is to obtain the measurement 1-2 frames before the opening of the valve. The maximum diameter of the left atrium is $12-16 \mathrm{~mm}$ in normal cats, with values greater than $16 \mathrm{~mm}$ indicating enlargement of the left atrium. In the authors' experience, 16-20 $\mathrm{mm}$ is consistent with mild dilatation, 20-25 mm with moderate dilatation, greater than $25 \mathrm{~mm}$ with severe dilatation.

Evidence of a thrombus may be noted on echocardiography in some cats with mild left atrial dilatation, while it may not be present in others with severe left atrial dilatation, There seems to be no relationship between the two. However, Rush et al. found that of 260 cats with HCM, 43 that developed ATE had a larger left atrium than those that developed heart failure or were preclinical [19]. Therefore, while the risk of ATE cannot be determined from the size of the left atrium, patients with a large left atrium need to be carefully monitored for the development of one. Therefore, antithrombotic therapy is recommended for all cats with moderate to severe left atrial dilatation. In addition, the presence of "smoke" within the left atrium on echocardiography suggests the existence of a prothrombotic condition, which may warrant antithrombotic therapy even in the absence of left atrial dilatation [19].

\section{Therapy}

Most treatments for myocardial disease in cats have been developed based on clinical experience rather than on data obtained from clinical trials.

Data is lacking regarding the effect of medical therapy on the development of CHF, ATE, and CSD in asymptomatic cats with HCM. Therefore, despite the controversy surrounding the use of medications in asymptomatic patients, clinicians worldwide prescribe beta-blockers, calcium channel blockers, ACE inhibitors, spironolactone, aspirin, and clopidogrel to cats with preclinical HCM.

Commonly prescribed drugs for the management of 
Table 1. Comparison between atenolol and diltiazem

\begin{tabular}{cll}
\hline & \multicolumn{1}{c}{ Atenolol } & \multicolumn{1}{c}{ Diltiazem } \\
\hline Drug class & Beta-blockers & Calcium channel blockers \\
\hline Main action & Negative inotropic & Anti-arrhythmic \\
& Negative chronotropic & Positive lusitropic \\
& Anti-ischemic & \\
& Anti-arrhythmic & \\
\hline Theoretical benefit & Relief of outflow tract obstruction & Heart rate control \\
& Heart rate control & Improved LV relaxation \\
& Antiarnythmic & \\
\hline
\end{tabular}

HCM include beta-blockers such as atenolol and calcium channel blockers such as diltiazem, with diltiazem being the most widely used. The differences in the mechanisms of action of these two drug classes are listed in Table 1 [20]. In particular, atenolol reduces myocardial oxygen demand, while diltiazem controls the heart rate and increases the amount of time allowed for filling of the the ventricles. Although diltiazem may improve relaxation of the heart muscle in some cats, this has not yet been proven conclusively. Atenolol is considered to have fewer side effects as compared to diltiazem, and is commonly used in HCM patients when there is an LVOTO (left ventricular outflow tract obstruction). This medication prevents tachycardia, improves ventricular filling capacity during diastole, and improves coronary blood flow. Along with its antiarrhythmic properties, atenolol inhibits myocardial hypertrophy in some individuals and helps alleviate clinical signs. The optimal dose of atenolol maintains the patient's heart rate at 120 160 BPM. However, attention should be paid to the possible development of hypotension and bradycardia. Its use is contraindicated in cases with CHF or ATE.

A cohort study conducted by Schober et al. evaluated the use of atenolol in asymptomatic patients [21]. The study included a total of 63 asymptomatic cats with HCM (42 in the atenolol group and 21 in the untreated control group). After five years of recording the mortality rate, no significant difference in the risk of death was found between the experimental group (24\%) and the control group (19\%). This study has caused much controversy regarding the use of atenolol in asymptomatic patients with HCM, in part because of a slight difference in the criteria used to assign patients to the two groups. At the start of the study, cats in the atenolol group had a significantly higher incidence of dynamic left ventricular outflow tract obstruction (DLVOTO), significantly more pronounced ventricular hypertrophy, a significantly higher grade heart murmur, higher peak velocity of the left ventricular outflow, and a significantly larger left atrium than cats in the control group $(p<0.05)$. In addition, the study size was relatively small, and it is difficult to compare survival rates between patients with and without DLVOTO. The study also did not include a method used to assess the quality of life of the cats. Therefore, although this study provides valuable information, clinicians continue to disagree about the use of atenolol in asymptomatic patients with HCM.

If the left atrial diameter exceeds $19 \mathrm{~mm}$ based on echocardiographic measurements, ACE inhibitors, antihypertensive drugs, and atenolol at a dose that does not interfere with contractility and does not cause bradycardia, are recommended. However, the use of atenolol and diltiazem together is not recommended because their combined use may result in bradycardia and hypotension.

Patients with HCM may develop acute CHF with pulmonary edema. If the patient is in the acute phase of CHF, treatment with "FONS" (furosemide, oxygen, nitroglycerin, and sedation) is indicated. This treatment is similar to the management of acute pulmonary edema in dogs, but in HCM patients, stress is also a very serious risk factor. Sedation is important, with butorphanol $(0.25$ $\mathrm{mg} / \mathrm{kg}$, IM or SC) commonly being used. Occasionally, acepromazine $(0.025-0.05 \mathrm{mg} / \mathrm{kg})$ may be administered in conjunction with butorphanol, but the patient's temperature should be maintained above $37.8^{\circ} \mathrm{C}$, and the arterial pressure above $100 \mathrm{mmHg}$, before considering its use. If the patient has a moderate volume of pleural effusion, this should be removed via thoracocentesis.

If the systolic arterial pressure measures less than $70 \mathrm{mmHg}$ and hypothermia or bradycardia-induced cardiogenic shock occurs, acute ischemic injury of the myocardium may develop and the chances of survival lessen. In these cases, treatment with dobutamine $(2.5 \mathrm{mcg} / \mathrm{kg} / \mathrm{min}$ IV) is warranted. The goal is a rectal temperature of $37.8^{\circ}$, arterial blood pressure of $90 \mathrm{mmHg}$ or more, and a heart rate of greater than 180 beats per minute. If the patient's condition does not improve, the dose of dobutamine may be increased to $5-10 \mathrm{mcg} / \mathrm{kg} / \mathrm{min}$. If the patient responds to treatment, the addition of an ACE inhibitor is indicated. Treatment with dobutamine should then be slowly tapered over a 23 hour period, ending at a dose that is $50 \%$ of the 
original.

For HCM patients with chronic CHF, the most basic drug therapy consists of furosemide (1-2 mg/ $\mathrm{kg}$, SID to BID) in combination with an ACE inhibitor. The use of spironolactone for the preservation of cardiac function and sparing of potassium may be considered, but it is not advised due to concerns regarding the development of anorexia and facial ulcerative dermatitis. Atenolol and diltiazem should be avoided in cases with CHF. However, if the patient is already taking either of these medications when (s)he experiences CHF and there is no evidence of cardiogenic shock, it is recommended that the dose of medication by reduced by $25 \%-50 \%$. If there is no evidence of DLVOTO and the patient remains stable for approximately one month after being diagnosed with $\mathrm{CHF}$, the atenolol dose may be increased again. For patients with chronic CHF, pimobendan $(\sim 0.25 \mathrm{mg} / \mathrm{kg}, \mathrm{BID}, \mathrm{PO})$ can be added for intensive therapy.

Recently, studies have been published that show positive results regarding the application of pimobendan to cases with HCM. Previously, the use of pimobendan was forbidden in cases with diastolic dysfunction. However, the potential for pimobendan to improve the diastolic function of the heart has been frequently discussed. According to Macgregor et al., the incidence of adverse events was only $3 \%$ in cats receiving pimobendan $(0.24 \mathrm{mg} / \mathrm{kg}$, BID) [22]. In particular, one study showed the effectiveness of pimobendan in the management of cases with HCM or HOCM over a 10 year period (from 2003 to 2013) [23]. This study divided the test subjects into two groups: pimo-

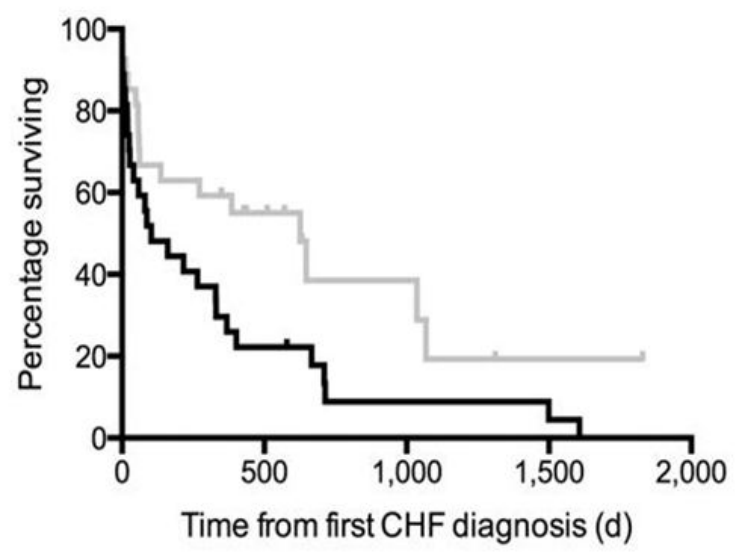

Fig. 4. Survival curve $(p=0.024)$ for cats with HCM or HOCM receiving pimobendan (gray line) versus the controls (black line). The mean survival times for the two groups were 626 days in the experimental group and 103 days in the control group. HCM, hypertrophic cardiomyopathy; HOCM, hypertrophic obstructive cardiomyopathy. Adapted from J Am Vet Med Assoc 2014;245:534-539, with permission of American Veterinary Medical Association [23]. bendan vs. control. The mean survival time for the cats in the pimobendan group was calculated to be 626 days, and the mean survival time for the cats in the control group was 103 days (Fig. 4). These results suggest that the use of pimobendan in cats with HCM or HOCM could result in a 6-fold increase in survival time. A cardiovascular drug, that has traditionally been used for the management of heart disease, digoxin, has now fallen out of favor. Famotidine $(2.5-5 \mathrm{mg} / \mathrm{kg}$, SID to BID) may be prescribed for anorexic patients. If a patient receiving diuretic monotherapy becomes azotemic, an ACE inhibitor is added. If the patient is already receiving combination therapy, it is necessary to adjust the dose of diuretic being administered.

Treatment for ATE caused by HCM aims to 1) prevent further clots from forming, 2) promote circulation to the ischemic tissue, 3) manage pain, and 4) dissolve existing emboli [24]. If the size of the left atrium measures greater than $19 \mathrm{~mm}$ and the echocardiogram shows "smoke" at the left atrium or a decrease in the contractile force of the chamber $(<0.2 \mathrm{~m} / \mathrm{s})$, the probability of the development of ATE is very high. In general, aspirin will be prescribed at less than $10 \mathrm{mg}$ per day and clopidogrel at $18.75 \mathrm{mg}$ per day. If clopidogrel is not available, aspirin is dosed once every three days at $80 \mathrm{mg} / \mathrm{cat}$. If more aggressive treatment is needed, therapy with either enoxaparin or dalteparin once or twice daily at a dose of $100 \mathrm{IU} / \mathrm{kg}$ is recommended.

Nevertheless, the use of antithrombotic drugs in asymptomatic cats with HCM remains controversial. In one pilot study, the administration of clopidogrel at $18.75 \mathrm{mg} / \mathrm{cat}$ SID to asymptomatic patients with HCM significantly reduced platelet aggregation without affecting the standard coagulation factor [25]. Therefore, the use of clopidogrel should be considered even in patients with asymptomatic HCM if the possibility of forming a blood clot exists.

In human medicine, anticoagulant medications are most commonly used for the management of thrombosis-related diseases [26, 27]. This principle is also extended to feline medicine, where aspirin is frequently administered. However, the use of aspirin as an anticoagulant medication for the management of ATE has been found to be less effective than the use of warfarin [28]. Clopidogrel is currently being considered as an alternative option to aspirin. A study from 2015 evaluating 75 cats that survived ATE associated with HCM, known as "FAT CAT", compared the survival rates of cats that received clopidogrel (18.75 $\mathrm{mg} / \mathrm{cat}$ PO q $24 \mathrm{~h}$ ) to those that received aspirin (81 $\mathrm{mg} / \mathrm{cat}$ PO q $72 \mathrm{~h}$ ) [29]. The cats in the clopidogrel group were shown to survive an average of 443 days while those in the aspirin group were shown to survive only 192 days (Fig. 5). This comparative study has led to 


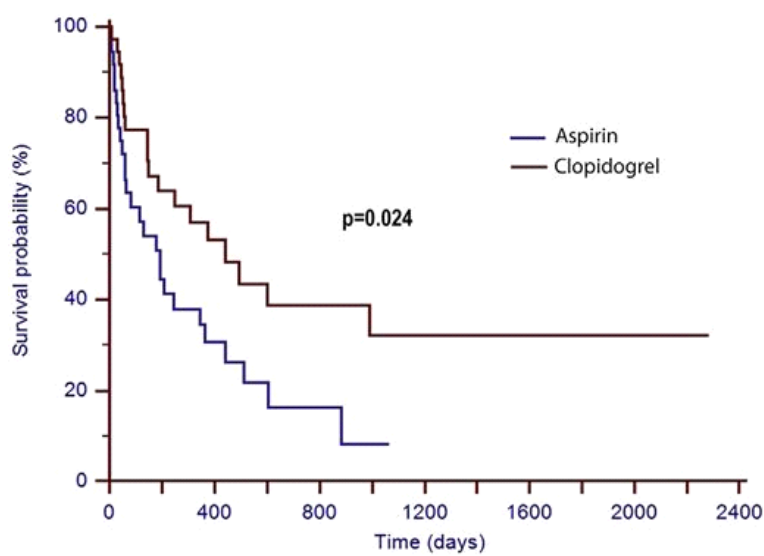

Fig. 5. Kaplan-Meier survival curves comparing aspirin and clopidogrel for the treatment of feline ATE. The group receiving clopidogrel survived an average of 443 days, while the group receiving aspirin survived an average of 192 days. ATE, arterial thromboembolism. Adapted from J Vet Cardiol 2015;17 Suppl 1:S306-S317, with permission of Elsevier [29].

a more favorable opinion regarding the use of clopidogrel in anticoagulant therapy.

In general, cats suffering from ATE are in pain, so analgesic treatment is appropriate. Aspirin $(70-81 \mathrm{mg} / \mathrm{cat}$, PO, EOD) can be used as an analgesic for cats and may also help prevent platelet aggregation [7, 30]. Opioids can potentially cause respiratory depression and bradycardia, but may be given to cats with cardiovascular disease because of their excellent analgesic properties. If used properly, opioids have little effect on myocardial contractility. Oxymorphone may be used at a dose of $0.05 \mathrm{mg} / \mathrm{kg}$ for moderate to severe pain [31] and butorphanol may be used at doses of 0.2 to $0.4 \mathrm{mg} / \mathrm{kg}$ for mild to moderate pain [31]. Both drugs may be given SC, IM, or IV q $4 \mathrm{~h}$ as needed to control pain.

Thrombolytic therapy is recommended to begin within 4 hours of the onset of clinical symptoms to maximize the possibility of reperfusion [7, 32]. Two drugs currently in use are streptokinase and tissue-plasminogen activator (t-PA) [33, 34]. However, both drugs possess a similar potential for causing reperfusion-related complications [33, 34].

Streptokinase is a nonspecific thrombolytic agent that activates circulating plasminogen. Plasminogen is converted to its active form, plasmin, which then breaks down fibrin. In cats with ATE, this mechanism can lead to thrombus dissolution, which restores perfusion to the ischemic tissue. However, because streptokinase is not specific for fibrin-bound plasminogen, its use may cause systemic fibrinolysis and clotting disorders. Therefore, the possibility of hemorrhage during treatment with streptokinase exists [33]. Streptokinase is administered at a dose of
$90,000 \mathrm{U}$ over 20 to 30 minutes, followed by a CRI of the medication at a dose of $45,000 \mathrm{U} / \mathrm{hr}$ over a 3 hour period [33].

Tissue-plasminogen activator is an enzyme that induces thrombolysis by converting fibrin-bound plasminogen to plasmin. Since t-PA is specific for plasminogen bound to the thrombus, its effect on circulating plasminogen is low and patients receiving this medication are less prone to bleeding than those receiving streptokinase [30, 34]. The total t-PA dose in cats is $1-10 \mathrm{mg} / \mathrm{kg}$ IV administered at 0.25-1.0 $\mathrm{mg} / \mathrm{kg} / \mathrm{hr}$ [34]. However, one report evaluating the use of t-PA in cats with ATE found that $70 \%$ of the complications leading to death were caused by lifethreatening metabolic acidosis and hyperkalemia [34]. Therefore, these potential complications should be kept in mind when treating cats with ATE, and the patients should be carefully monitored using a combination of clinical assessment, biochemical analysis, and ECG monitoring.

Thrombolysis with interventional procedures using an arterial catheter may also be considered for the treatment of cats with ATE. When treatment is initiated within a few hours of the onset of clinical signs, immediate results may be seen but the risk of recurrent embolism remains [35]. Therefore, additional therapies should be considered.

\section{Conclusion}

The average survival time of asymptomatic cats with HCM was found to be 1,129 days, and the average survival time of cats with HCM and CHF was 563 days [19]. The larger the size of the left atrium, and the older the patient with HCM, the worse the prognosis. These patients with HCM cannot be cured, and treatment should be aimed at prolonging their survival times and improving their quality of life. For patients with asymptomatic HCM, reevaluation is recommended every 6-12 months. For cats receiving medical therapy (diuretics, ACE inhibitors, etc.), periodic imaging of the heart and lungs (radiography and echocardiography) and regular monitoring of kidney function (analysis of blood and urine) are required.

Given the poor prognosis of cats with ATE and the reported recurrence rate of $24 \%-75 \%$ [8, 36, 37], this condition is a critical one. Typically, hospitalization for up to 10 days is required, and the average length of stay for cats that survive is approximately 2 days [15]. There are many different ways to assess the severity of ATE in cats, but the simplest measurement indicating a poor prognosis is a low rectal temperature $[15,38]$. The rectal temperature of patients who died within 24 hours of the onset of clinical signs averaged $36^{\circ} \mathrm{C}$, whereas that of patients who survived beyond 24 hours averaged $37.8^{\circ} \mathrm{C}$. 


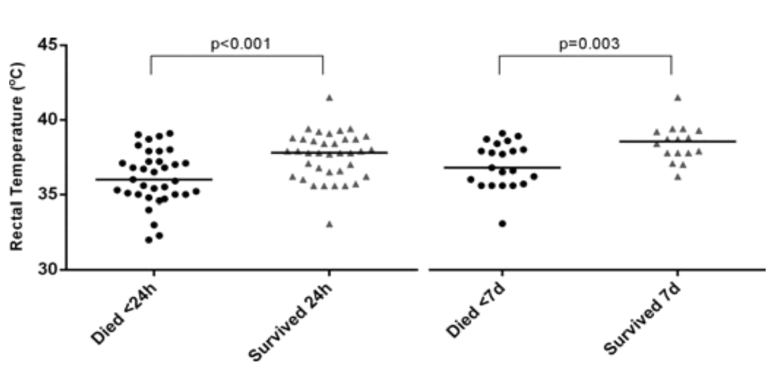

Fig. 6. Scatter plot of rectal temperatures in patients with ATE. The mean rectal temperature of patients who died within 24 hours of the onset of clinical signs was $36^{\circ} \mathrm{C}$, whereas that of patients who survived beyond 24 hours was $37.8^{\circ} \mathrm{C}$. The mean rectal temperature of patients who died within 7 days was $36.8^{\circ} \mathrm{C}$, whereas that of patients who survived beyond 7 days was $38.6^{\circ} \mathrm{C}$. ATE, arterial thromboembolism. Adapted from J Vet Intern Med 2014;28:102-108 [15].

The rectal temperature of patients who died within 7 days averaged $36.8^{\circ} \mathrm{C}$, whereas that of patients who survived beyond 7 days averaged $38.6^{\circ} \mathrm{C}$ (Fig. 6). In addition, the prognosis was improved when the ATE affected only one hind limb [15].

Long-term survival in cats following ATE depends largely on the existence of $\mathrm{CHF}$ at the time of diagnosis. Although ATE in most feline patients is caused by HCM, the average survival time in cats with ATE and concurrent CHF is approximately 77 days [6]. The average survival time of cats without signs of CHF is 223 days, with some of these cats surviving for an additional 2 years [6]. In addition, the development of moderate to severe azotemia and hematochezia are negative prognostic indicators that suggest the presence of systemic thromboembolic disease.

Survival following ATE can also lead to such complications such as tissue necrosis, infection, and hind limb atrophy. The development of tissue necrosis or infection can be difficult to manage and may result in the need for amputation.

ATE is a very serious disease in cats and is usually caused by the presence of heart disease, namely HCM. Therefore, ATE patients with underlying heart disease should be monitored closely for the development of CHF.

\section{ORCID}

Donghyun Han, http://orcid.org/0000-0003-3258-244X

Dong-In Jung, http://orcid.org/0000-0002-5116-6006

\section{References}

1. Paige CF, Abbott JA, Elvinger F, Pyle RL. Prevalence of cardiomyopathy in apparently healthy cats. J Am Vet Med
Assoc 2009;234:1398-1403.

2. Rishniw M, Thomas WP. Dynamic right ventricular outflow obstruction: a new cause of systolic murmurs in cats. J Vet Intern Med 2002;16:547-552.

3. Lefbom BK, Rosenthal S, Tyrell WDJ, Saunders TG, Ferguson MJ, Rush JE, Lesser MB. Severe hypertrophic cardiomyopathy in 10 young Ragdoll cats. J Vet Intern Med 2001;15: 308.

4. Oyama MA. Cardiogenic pulmonary edema. In: Drobatz KJ, Hopper K, Rozanski E, Silverstein DC (eds.). Textbook of small animal emergency medicine. Hoboken,: John Wiley \& Sons; 2019. p. 242-246.

5. Gann D, Segall PH, Samet P, Korn D. Cardiac-pulmonary edema and low pulmonary capillary wedge pressure. South Med J 1979;72:1413-1415.

6. Smith SA, Tobias AH, Jacob KA, Fine DM, Grumbles PL. Arterial thromboembolism in cats: acute crisis in 127 cases (1992-2001) and long-term management with low-dose aspirin in 24 cases. J Vet Intern Med 2003;17:73-83.

7. Rush JE. Therapy of feline hypertrophic cardiomyopathy. Vet Clin North Am Small Anim Pr 1998;28:1459-1479.

8. Laste NJ, Harpster NK. A retrospective study of 100 cases of feline distal aortic thromboembolism: 1977-1993. J Am Anim Hosp Assoc 1995;31:492-500.

9. Fox PR, Keene BW, Lamb K, Schober KA, Chetboul V, Luis Fuentes V, Wess G, Payne JR, Hogan DF, MotsingerReif A, Haggstrom J, Trehiou-Sechi E, Fine-Ferreira DM, Nakamura RK, Lee PM, Singh MK, Ware WA, Abbott JA, Culshaw G, Riesen S, Borgarelli M, Lesser MB, Van Israel N, Cote E, Rush JE, Bulmer B, Santilli RA, Vollmar AC, Bossbaly MJ, Quick N, Bussadori C, Bright JM, Estrada AH, Ohad DG, Fernandez-Del Palacio MJ, Lunney Brayley J, Schwartz DS, Bove CM, Gordon SG, Jung SW, Brambilla P, Moise NS, Stauthammer CD, Stepien RL, Quintavalla C, Amberger C, Manczur F, Hung YW, Lobetti R, De Swarte M, Tamborini A, Mooney CT, Oyama MA, Komolov A, Fujii Y, Pariaut R, Uechi M, Tachika Ohara VY. International collaborative study to assess cardiovascular risk and evaluate long-term health in cats with preclinical hypertrophic cardiomyopathy and apparently healthy cats: The REVEAL study. J Vet Intern Med 2018;32:930-943.

10. Maron MS, Olivotto I, Betocchi S, Casey SA, Lesser JR, Losi MA, Cecchi F, Maron BJ. Effect of left ventricular outflow tract obstruction on clinical outcome in hypertrophic cardiomyopathy. N Engl J Med 2003;348:295-303.

11. Hamada M, Ikeda S, Shigematsu Y. Advances in medical treatment of hypertrophic cardiomyopathy. J Cardiol 2014; 64:1-10.

12. Litster AL, Buchanan JW. Vertebral scale system to measure heart size in radiographs of cats. J Am Vet Med 
Assoc 2000;216:210-214.

13. Fox PR, Rush JE, Reynolds CA, Defrancesco TC, Keene BW, Atkins CE, Gordon SG, Schober KE, Bonagura JD, Stepien RL, Kellihan HB, Macdonald KA, Lehmkuhl LB, Nguyenba TP, Sydney Moise N, Lefbom BK, Hogan DF, Oyama MA. Multicenter evaluation of plasma N-terminal probrain natriuretic peptide (NT-pro BNP) as a biochemical screening test for asymptomatic (occult) cardiomyopathy in cats. J Vet Intern Med 2011;25:1010-1016.

14. Sangster JK, Panciera DL, Abbott JA, Zimmerman KC, Lantis AC. Cardiac biomarkers in hyperthyroid cats. J Vet Intern Med 2014;28:465-472.

15. Borgeat K, Wright J, Garrod O, Payne JR, Fuentes VL. Arterial thromboembolism in 250 cats in general practice: 2004-2012. J Vet Intern Med 2014;28:102-108.

16. Green HW, Hogan DF. Suspected iatrogenic paradoxical embolization in a cat. J Am Anim Hosp Assoc 2005; 41: 193-197.

17. Atkins CE, Gallo AM, Kurzman ID, Cowen P. Risk factors, clinical signs, and survival in cats with a clinical diagnosis of idiopathic hypertrophic cardiomyopathy: 74 cases (19851989). J Am Vet Med Assoc 1992;201:613-618.

18. Peterson EN, Moise NS, Brown CA, Erb HN, Slater MR. Heterogeneity of hypertrophy in feline hypertrophic heart disease. J Vet Int Med 1993;7:183-189.

19. Rush JE, Freeman LM, Fenollosa NK, Brown DJ. Population and survival characteristics of cats with hypertrophic cardiomyopathy: 260 cases (1990-1999). J Am Vet Med Assoc 2002;220:202-207.

20. Fox PR, Schober KA. Management of asymptomatic (occult) feline cardiomyopathy: challenges and realities. J Vet Cardiol 2015; 17:S150-S158.

21. Schober KE, Zientek J, Li X, Fuentes VL, Bonagura JD. Effect of treatment with atenolol on 5-year survival in cats with preclinical (asymptomatic) hypertrophic cardiomyopathy. J Vet Cardiol 2013;15:93-104.

22. Macgregor JM, Rush JE, Laste NJ, Malakoff RL, Cunningham SM, Aronow N, Hall DJ, Williams J, Price LL. Use of pimobendan in 170 cats $(2006-2010)$. J Vet Cardiol 2011;13:251-260.

23. Reina-Doreste Y, Stern JA, Keene BW, Tou SP, Atkins CE, DeFrancesco TC, Ames MK, Hodge TE, Meurs KM. Casecontrol study of the effects of pimobendan on survival time in cats with hypertrophic cardiomyopathy and congestive heart failure. J Am Vet Med Assoc 2014;245:534-539.

24. Rodriguez DB, Harpster N. Aortic thromboembolism associated with feline hypertrophic cardiomyopathy. Comp Cont Educ Pr 2002;24:478-483.
25. den Toom ML, van Leeuwen MW, Szatmari V, Teske E. Effects of clopidogrel therapy on whole blood platelet aggregation, the plateletworks ${ }^{\circledR}$ assay and coagulation parameters in cats with asymptomatic hypertrophic cardiomyopathy: a pilot study. Vet Q 2017;37:8-15.

26. Deedwania PC, Huang GW. Role of emerging antithrombotic therapy in the prevention of cardioembolic complications in patients with atrial fibrillation. Am J Cardiovasc Drugs 2011;11:265-275.

27. Freeman WD, Aguilar MI. Prevention of cardioembolic stroke. Neurotherapeutics 2011;8:488-502.

28. Harpster NK, Baty CJ. Warfarin therapy of the cat at risk of thromboembolism. In: Bonagura JD (ed.). Kirk's current veterinary therapy XII small animal practice. Philadelphia: WB Saunders; 1995. p. 868-873.

29. Hogan DF, Fox PR, Jacob K, Keene B, Laste NJ, Rosenthal S, Sederquist K, Weng HY. Secondary prevention of cardiogenic arterial thromboembolism in the cat: the double-blind, randomized, positive-controlled feline arterial thromboembolism; clopidogrel vs. aspirin trial (FAT CAT). J Vet Cardiol 2015;17:S306-S317.

30. Behrend EN, Grauer GF, Greco DS. Feline hypertrophic cardiomyopathy, Part 3. Feline Pract 1997;25:22-25.

31. Carroll GL. Analgesics and pain. Vet Clin North Am Small Anim Pract 1999;29:701-717.

32. Rentrop KP. Thrombolytic therapy in patients with acute myocardial infarction. Circulation 1985;71:627-631.

33. Killingsworth CR, Eyster GE, Adams T, Bartlett PC, Bell TG. Streptokinase treatment of cats with experimentally induced aortic thrombosis. Am J Vet Res 1986;47:13511359.

34. Pion PD, Kittleson MD. Therapy for feline aortic thromboembolism. In: Kirk RW, Bonagura JD (eds.). Current veterinary therapy $\times$ small animal practice. Philadelphia: WB Saunders 1989;295-302.

35. Buchanan JW, Baker GJ, Hill JD. Aortic embolism in cats: prevalence, surgical treatment and electrocardiography. Vet Rec 1966;79:496-505.

36. Fox PR. Evidence for or against efficacy of beta-blockers and aspirin for management of feline cardiomyopathies. Vet Clin North Am Small Anim Pract 1991;21:1011-1022.

37. Moore KE, Morris N, Dhupa N, Murtaugh RJ, Rush JE. Retrospective study of streptokinase administration in 46 cats with arterial thromboembolism. J Vet Emerg Crit Care 2000;10:245-257.

38. Smith SA, Tobias AH. Feline arterial thromboembolism: an update. Vet Clin Small Anim Pract 2004;34:1245-1271. 